\title{
Immediate Loading of Onepiece Root Form Implant: A Case Report with 3 Years' Follow-up
}

\author{
${ }^{1}$ Shivaprasad Bilichodmath, ${ }^{2}$ Shilpa Shivanand ${ }^{3}$ Navnita Singh
}

\section{ABSTRACT}

Aim: The aim of this case report was to evaluate the clinical performance of a onepiece implant in a partially edentulous anterior mandible.

Introduction: Osseointegration being an accepted and welldocumented concept, attention is nowdirected toward simplification of the mechanical design of implants and toward achieving biomechanical success.

Case report: This case report demonstrates the immediate loading of one piece root form implant in esthetically concern patient. Patient came to the clinic with the chief complain of missing lower front teeth and wanted replacement of the same.

Conclusion: A single stage, one piece implant placement with immediate loading provides a good soft tissue healing and minimal postoperative discomfort to the patient.

Clinical significance: One piece root form implants is a reliable treatment protocol in esthetically concerned patients.

Keywords: Immediate provisonalization, Minimally invasive implant placement, Single piece implant.

How to cite this article: Bilichodmath S, Shivanand S, Singh N. Immediate Loading of Onepiece Root Form Implant: A Case Report with 3 Years' Follow-up. J Health Sci Res 2016;7(1):16-18.

Source of support: Nil

Conflict of interest: None

\section{INTRODUCTION}

Over the past two decades, implant treatment has become one of the first options for the prosthetic rehabilitation of edentulous and partially edentulous jaws. The actual Branemark concept consists of a two-piece dental implant designed to be used in a two-stage treatment procedure. After raising a soft tissue flap, the implant is placed into the bone, which is followed by repositioning to cover the implant during healing. Following a healing period, a new flap is raised, and a transmucosal abutment is attached to the implant to allow the prosthesis to be connected. ${ }^{1}$ The conventional two-piece implant (TPI) design feature has the implant-abutment connection, rendering

\footnotetext{
${ }^{1}$ Reader, ${ }^{2,3}$ Postgraduate Student

${ }^{1-3}$ Department of Periodontology, RajaRajeswari Dental College \& Hospital, Bengaluru, Karnataka, India

Corresponding Author: Shivaprasad Bilichodmath, Reader Department of Periodontology, RajaRajeswari Dental College \& Hospital, Bengaluru, Karnataka, India, Phone: +919900511071 e-mail: drspbmath@gmail.com
}

the design with a weak link in the entire assembly. A smooth transition from the root analogue to the crown analogue overcomes this drawback of the two-piece (split) implant. ${ }^{2,3}$ Such a seamless transition of implant to abutment is design advantage offered by one-piece implant (OPI), which actually mimics the natural tooth in its construction and also offers many advantages, namely, strong unibody design, no split parts, singlestage surgery with either flap or flapless approach, and simple restorative technique. ${ }^{4}$ Thus, the unibody design of OPI eliminates the fixture abutment interface (microgap) and mimics natural tooth with seamless transition of the radicular unit to coronal unit. Following are the indications of OPIs: (1) Immediate implant placement in fresh extraction socket and (2) narrow edentulous spaces. Advantages of OPIs include: (1) The OPI has more mechanical strength because the implant cross section is solid as compared to TPI. ${ }^{5}$ (2) As OPI has no microgap between the implant and the abutment, it shows reduced marginal bone loss. Thus, the loss of alveolar bone around the implants is minimized as it cannot harbor bacteria. ${ }^{6}$ (3) OPI reduces the requirement of multiple surgical procedures and prosthetic components, thereby reducing inventory (abutments, impression copings, implant analogs, etc.) and cost. (4) No loosening or fracture of the abutment screw. ${ }^{7}(5)$ The final crown margins preparation can be controlled in a rapid and easy manner. (6) The OPI follows the conventional crown and bridge procedure. Disadvantages of OPIs include: (1) It cannot be used in case of tilted abutments in which the tilt is more than 10 to $15^{\circ}$ aas heavy occlusal loads are applied in posterior edentulous areas over the restoration immediately. ${ }^{8}$ (2) it allows only the use of a knife-edge margin for the final prosthesis as providing chamfer of shoulder to final restoration leads to structural weakness in the final restoration. ${ }^{9}$ An OPI allows a minimally invasive transmucosal flapless placement, and it limits the requirement of hard tissue grafting procedures. The present case study evaluated the clinical performance of an OPI in a partially edentulous anterior mandible.

\section{CASE REPORT}

A 31-year-old female patient presented to the Sparsh Dental Clinic, Bengaluru, seeking replacement of a missing tooth in relation to tooth number 31 and 41 . The patient was 
well-informed of the immediate loading as well as the twostage protocol and chose the former method. A presurgical radiographic evaluation was carried out with panoramic radiographs and digital intraoral radiographs. The ridge width was evaluated by ridge mapping and the implant size $(2.8 \times 13 \mathrm{~mm})$ was selected by using the expanders in the kit as dummy implants on the diagnostic casts. The surgical procedure was performed under local anesthesia. Incision was made at the crest of the alveolar ridge and the flaps were reflected minimally to analyze the width. An initial drill was used for site preparation to give needlepoint accuracy for position, angle, and depth along with the use of copious saline irrigation. Subsequently, the drill size of $2.2 \mathrm{~mm}$ was used up to $11.5 \mathrm{~mm}$ depth. The drill passed through the cortical bone and then the cancellous bone. A depth gage was used to assess the integrity of the bone in all the five dimensions. The implant was placed using a carrier and rotated clockwise. For complete seating of the implant, a hex driver with a ratchet wrench was used. Primary stability was found to be $40 \mathrm{Ncm}$ and the final seating of the implant was checked using radiovisiography (Fig. 1). The provisional acrylic resin restoration was fabricated and care was taken to achieve a smooth contour of the provisional tooth, to avoid irritation of the soft tissue, as well as to condition the soft tissue profile. The provisional crown was placed out of contact proximally and occlusally; it was temporarily cemented. The patient

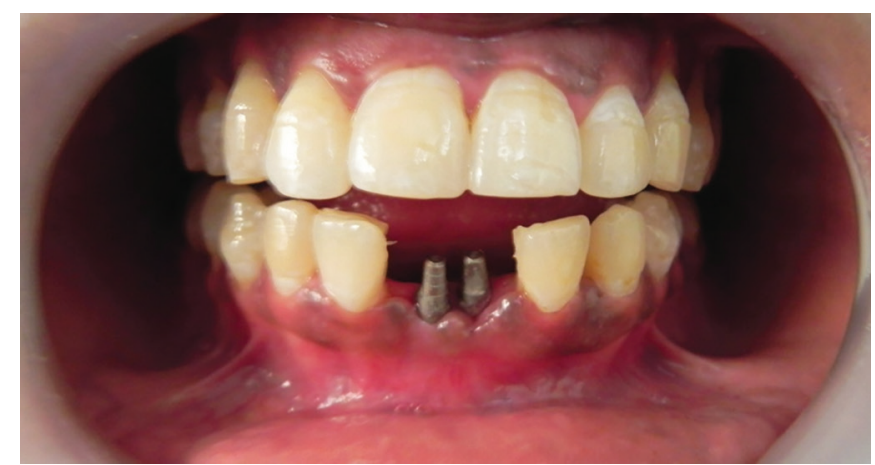

Fig. 1: One-piece implant in position

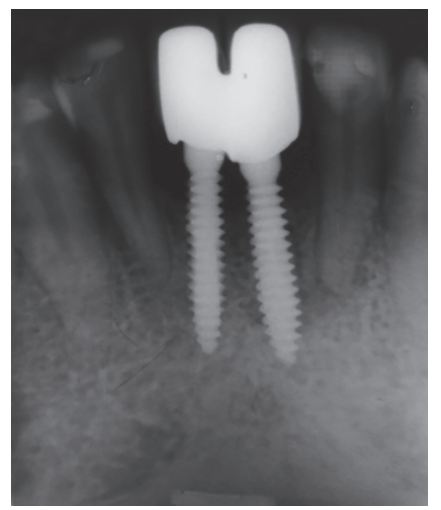

Fig. 3: lopar showing the OPI in position with the permanent restoration was instructed to avoid directly biting on the provisional restoration and to consume easily chewable food for 2 months. The patient experienced minimal postoperative pain and no swelling. Complete soft tissue healing was achieved during the first 2 weeks of implant placement, following which, procedures required for permanent restoration were performed using addition silicone impression material. Finally, porcelain fused to metal (PFM) crown was placed in position using glass ionomer cement as luting agent (Figs 2 and 3). The radiographic crestal bone loss, by the end of 3 years of functional loading, was found to be negligible (Figs 4 and 5).

\section{DISCUSSION}

Immediate loading of dental implants is becoming a widespread therapeutic procedure for the rehabilitation of patients with edentulous jaws. A review by Prithviraj et $\mathrm{a}^{10}$ on an OPI concluded that most of the surgeons preferred delayed placements with immediate loadings of OPIs. It was reported that the use of an undersized form drill, in combination with, a conical implant design, could lead to a higher initial stability than that, which was seen with conventional implants. 10 As far as the surgical protocol is considered, less patient discomfort (less pain, swelling, analgesic dose) was observed in flapless procedures than in surgeries with flap. Moreover, it took fewer appointments and less surgical time. Also, blood

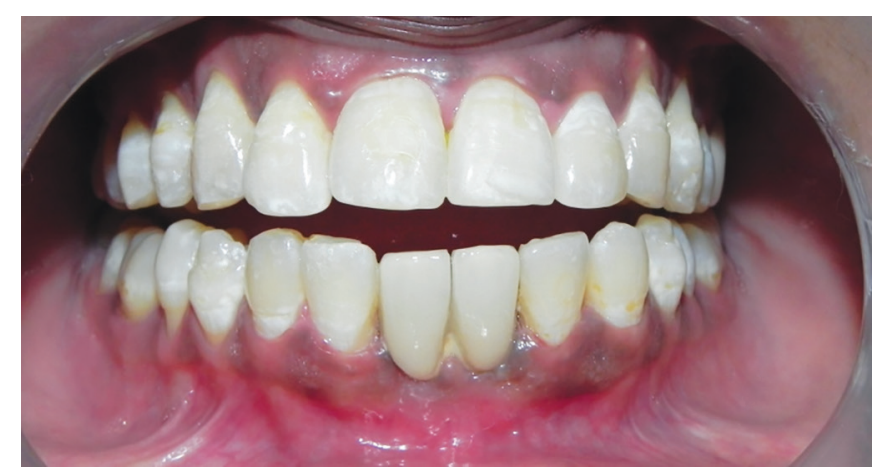

Fig. 2: Porcelain fused to metal crown in position

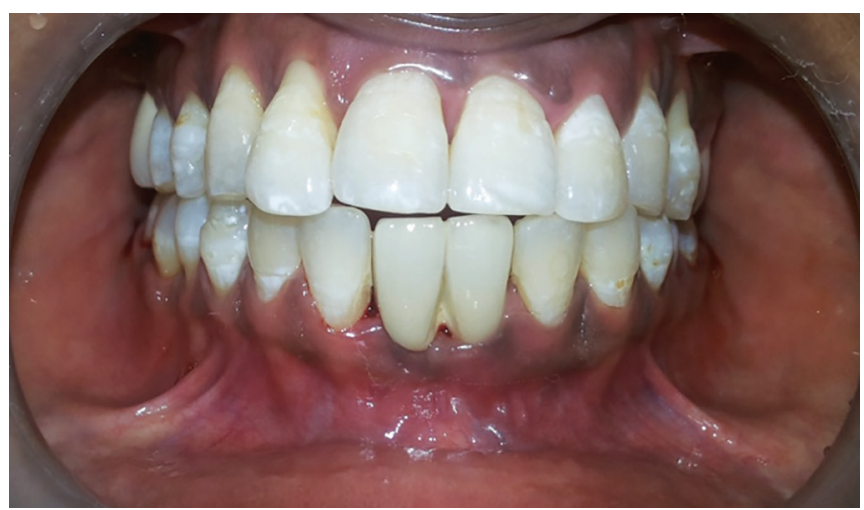

Fig. 4: Three years' follow-up 


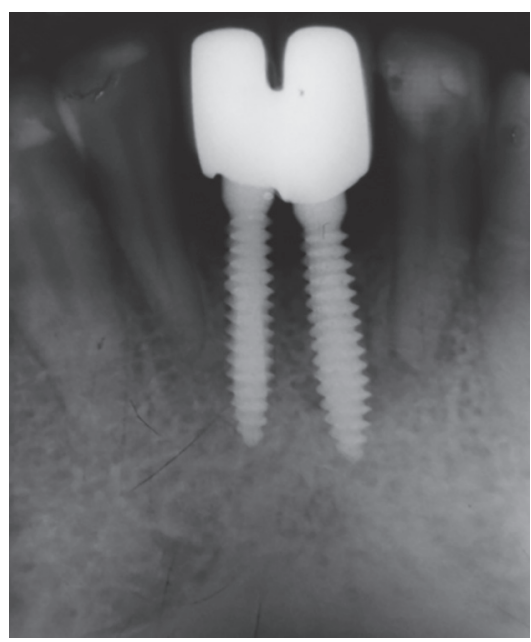

Fig. 5: Three years' follow-up lopar

supply to the underlying bone was maintained; however, despite these advantages, the flapless technique also has several potential shortcomings. It becomes difficult for the surgeon to visualize the anatomical landmarks and vital structures during implant placement. The potential for thermal trauma to the bone is greater due to limited external irrigation during preparation of the osteotomy site. Another disadvantage of the flapless procedure is the possibility of contamination of the implant surface or the deposition of epithelial or connective cells in the hole in the bone, which can interfere with osseointegration. ${ }^{11}$ Although flapless implant placement is considered to be a blind surgical procedure, there is a learning curve with every surgical procedure, after which it becomes routine. Clinical studies by Bashutski et $\mathrm{al}^{12}$ and Froum et $\mathrm{al}^{13}$ found no difference between flapless surgeries and traditional flap procedures on single tooth implants with higher survival rates, and stable marginal bone and pocket depths in both the groups. There are many advantages for the patients as well as for the surgeons, since in this procedure bleeding is minimal, less time-consuming as implant placement is expedited, and as there is no need to place and remove sutures. The OPI design eliminates the need for placing healing collars and it makes it possible to avoid manipulation of the soft tissue portion after initial healing. The implant abutment junction in a TPI design constitutes a structural weakness that may complicate the procedure. ${ }^{14}$ The primary stability of 40 to $80 \mathrm{Ncm}$ is completely satisfactory to allow immediate loading or at least to provide immediate restoration. Marco et $a{ }^{15}$ found no statistically significant difference between immediate and one-staged restored small-diameter implants, with regard to implant survival, mean marginal bone loss, and pocket depth in clinical trials, which were carried out over 3 years. In this case report, we have reported the immediate loading of OPI, which reduces the waiting period of patient for permanent restoration.

\section{CONCLUSION}

A single-stage OPI placement with immediate loading provides good, soft tissue healing and minimal postoperative discomfort to the patient. In this case report, immediate loading of an anterior single-piece implant radiographically revealed a stable marginal bone around the implant, with a maintainable peri-implant soft tissue integrity and edentulous space, both occusally and mesiodistally. Thus, within the limits of this case report, a single-stage OPI placement, with immediate loading, provides greater advantages with high acceptance by the surgeons as well as the patients.

\section{REFERENCES}

1. Swart LC, van Niekerk DJ. Simplifying the implant treatment for an unrestorable premolar with a one-piece implant: a clinical report. J Prosthet Dent 2008;100:81-85.

2. O'Mahony A, et al. Design features that may influence bacterial Plaque retention: a retrospective analysis of failed implants. Quintessence Int 2000;31:249-256.

3. Tsuge T, Hagiwara Y, Matsumura H. Marginal Fit and Microgaps of Implant-abutment Interface with Internal Antirotation Configuration. Dent Mat J 2008;27(1):29-34.

4. Daniel BR, et al. Clinical experience with one-stage nonsubmerged dental implants. Adv Dent Res 1999;13:153-161.

5. Siepenkothen T. Clinical performance and radiographic evaluation of a novel single-piece implant in a private practice over a mean of seventeen months. J Prosthet Dent 2007; 97: S69-S78.

6. Broggini N, McManus LM, Hermann JS, et al. Persistent acute inflammation at the implant abutment interface. J Dent Res 2003; 82:232-237.

7. Stephen M. Parel, Sterling R. Schow. Early Clinical Experience with a New One-Piece Implant System in Single Tooth Sites. J Oral Maxillofac Surg 2005; 63(9 S2):2-10.

8. Hahn J. One piece root form implants: a return to simplicity. J. Oral implantol 2005;2:77-84.

9. Sohn D, et al. Retrospective Multicenter Analysis of Immediate Provisionalization Using One- Piece Narrow-Diameter (3.0-mm) Implants. Int J Oral Maxillofac Implants 2011;26: 163-168.

10. Prithviraj DR, Onepiece implants: placement timing, surgical technique, loading protocol, and marginal bone loss J Prosthodont 2013;22(3):237-244.

11. Fortin T, Bosson JL, Isidori M, et al. Effect of flapless surgery on pain experienced in implant placement using an imageguided System. Int J Oral Maxillofac Implants 2006;21: 298-304.

12. Bashutski Jill D. The Effect of Flapless Surgery on SingleTooth Implants in the Esthetic Zone: a Randomized Clinical Trial J Periodont 2013.

13. Froum Stuart J. Survival rate of one piece dental implants placed with flapless or flap protocol - A Randomized, controlled study; 12 months results. Int J Periodont Dent 2011;31:591-601.

14. Hahn J. Singlestage, immediate loading, and flapless surgery J Oral Implantol 2000;26:193-98.

15. Degidi M, Nardi D, Piattelli A. Immediate Versus OneStage Restoration of Small Diameter Implants for a Single Missing Maxillary Lateral Incisor: a 3 years Randomized Clinical Trial J Periodontol 2009;80:139-398. 